\title{
Los fondos de inversión inmobiliaria y la producción privada de vivienda en Santiago de Chile: ¿Un nuevo paso hacia la financiarización de la ciudad?
}

\author{
Rodrigo Andrés Cattaneo Pineda \\ CREDAL - Centre de Recherche et de Documentation sur l'Amérique Latine - UMR \\ 7227.
}

RESUMEN | A pesar de la influencia en el debate académico de la hipótesis de la relación entre la globalización económica y la emergencia de un nuevo modelo de metrópolis, pocas publicaciones han abordado el análisis de los mecanismos concretos de articulación de estas dos esferas. El objetivo de este artículo es el estudio del papel jugado por una categoría de actores del mercado de capitales -los fondos de inversión inmobiliaria chilenos- en la producción de los espacios residenciales de Santiago. Por medio del examen de su portafolio de colocaciones habitacionales, se subraya su importancia en el financiamiento de la edificación privada de vivienda. Para definir estos programas, los fondos han privilegiado las tipologías y las localizaciones que se adecuan mejor a sus temporalidades y a sus lógicas de funcionamiento. De este modo, han tenido influencia directa sobre la verticalización de la ciudad, y en los procesos de renovación de los barrios céntricos. Vemos en la difusión de las exigencias del mercado de capitales, así como de sus técnicas de gestión de activos, una nueva faceta del fenómeno de "financiarización" de las grandes metrópolis en el sector habitacional.

PAlABRAS ClAVE | Servicios financieros, economía urbana, vivienda.

\begin{abstract}
Despite the influence in the scientific debate of the bypothesis of an interaction between economic globalization and the development of a new metropolitan model, few studies have handled the analysis of the concrete forms of articulation between these two spheres. The main purpose of this paper is to appraise the contribution of one form of capital market operators -Chilean real estate investment trusts-in the making of housing spaces in Santiago. Based on the examination of their residential assets, this article shows their growing role in the funding of private housing. They seem to favor housing models and localizations that suitably match up the timings and policies of their internal functioning. Consequently, it appears they have direct responsibility on the verticalization of the skyline and on the renovation of central neighborhoods. In the diffusion of the requirements the capital markets in the housing sector and in its asset management tools we see a new facet of the progressive "finantiarization" of big metropolis.
\end{abstract}

KEY WORDS | Financial services, urban economy, housing.

Recibido el 8 de enero de 2011, aprobado el 5 de mayo de 2011.

Correspondencia: CREDAL - Centre de Recherche et de Documentation sur l'Amérique Latine - UMR 7227, 52, rue de Crimée-75019, Paris, Francia; e-mail: rcattaneo@univ-paris8.fr 


\section{Introducción}

La tesis de un vínculo estrecho entre la globalización de las cadenas productivas y la reconfiguración espacial de las metrópolis mundiales es una de las ideas que ha estructurado el debate académico sobre la ciudad en los últimos tres decenios. Ahondando las conclusiones de Peter Geoffrey Hall (1966) sobre las funciones de comando de las "world cities", John Friedmann y Goetz Wolff (1982) renovaron la agenda científica con un artículo cardinal sobre la internacionalización del capital y la reorganización de las grandes metrópolis. La evolución de los procesos de urbanización y de la morfología urbana se veía explicada por el rol protagónico de las ciudades como espacios de regulación de la globalización (Veltz, 1996). Pero la primacía acordada a los factores estructurales y a la temática de la mundialización en el estudio del cambio urbano dio un énfasis quizás excesivo a la definición y a la descripción de la "ciudad global", en detrimento de un estudio de los procesos, actores y mecanismos que permiten la transición a un nuevo régimen urbano. Pocas publicaciones se han interesado en los vínculos concretos entre la transformación de los paradigmas económicos y la fabricación de los espacios urbanos (Grant y Nijman, 2004). A pesar de los numerosos indicios que respaldan este postulado, el carácter privado de las informaciones sobre la gestión interna de las empresas ha generalmente impedido circunscribir la acción de los agentes económicos, así como reconstituir sus estrategias de inversión a escala metropolitana. Para suplir esta carencia, este artículo se ha dado como objetivo el análisis de los lazos crecientes entre los mercados financieros y el sector inmobiliario, y de su impacto sobre el financiamiento de la inversión y sobre la morfología y los paisajes metropolitanos de la ciudad de Santiago.

La puesta en práctica precoz de las "reformas estructurales" hizo de Chile un campo de observación privilegiado para el seguimiento de los efectos de la liberalización económica (Velut, 2007). No es de extrañar, por ende, que Santiago haya ocupado una posición central en el debate sobre el modelo de la ciudad latinoamericana en el contexto del ciclo neoliberal (Meyer y Bahr, 2001; De Mattos, 2003; Borsdorf, Hidalgo y Sánchez, 2007). Su ejemplo parecía confirmar la hipótesis de una relación estrecha entre economía y formas urbanas. En efecto, la liberalización del mercado de suelo urbano durante el periodo 1978-1985 fue un factor clave en la explicación de los cambios en el proceso de fabricación de la ciudad y de las consiguientes transformaciones del tejido urbano. Nuevos actores -empresas constructoras, promotores inmobiliarios, corredores de bienes raíces- emergieron al alero de la reforma, substituyéndose progresivamente al sector público como principal referente en el campo de la vivienda (Sabatini, 2000).

A nuestro entender, el sistema de producción de la ciudad conoce un nuevo ciclo de transformaciones a contar de mediados de los años noventa, y sobre todo a partir de la primera reforma del mercado de capitales (2001). Nuevos mecanismos y dispositivos legales buscaron favorecer su liquidez y su profundidad, y simplificar el acceso a nuevos canales de financiamiento para las empresas emergentes (Cifuen- 
tes, Desormeaux y González, 2002). Se intensifica, entonces, un movimiento multiforme de acoplamiento entre el campo de la finanza y la promoción inmobiliaria: participación creciente de los inversionistas institucionales, apertura del capital de los principales desarrolladores (Salfacorp S.A. en 2004, seguida por Pazcorp S.A. y Socovesa S.A.), creación o adquisición de firmas inmobiliarias por holdings financieros (compra de la Constructora Moller y Pérez-Cotapos por el fondo de inversión Citigroup Venture Capital International en 2007; creación de filiales especializadas por los grupos Security -1996-, Penta -2000- y Banmerchant -2002-). La diversificación de las fuentes de financiamiento de la industria de la vivienda ha sido así un formidable acelerador de las dinámicas metropolitanas. En este articulo hemos privilegiado el examen del rol inédito, pero medular, de los fondos inmobiliarios mixtos y de desarrollo ${ }^{1}$ en la producción habitacional. En un sector caracterizado por su heterogeneidad y su alto grado de atomización, estos fondos brillan por su participación creciente en el financiamiento de la construcción privada. Los recursos administrados por estos vehículos, especialmente después de la creación de los primeros fondos dedicados al desarrollo de proyectos en 2004, les han permitido asociarse a programas habitacionales equivalentes a por lo menos el $12,8 \%$ de la oferta metropolitana de vivienda ${ }^{2}$.

Si la influencia de estos fondos y de otros operadores financieros se ha hecho también sentir con fuerza en otros rubros, como en equipamientos relacionados con el consumo (shopping malls, supermercados, strip centers, etc.) o en la oferta de oficinas corporativas, hemos privilegiado la cuestión de la vivienda por su peso económico ${ }^{3}$ y por su importancia en la configuración física de la ciudad. La revisión de sus memorias anuales y cartolas trimestrales, asociada a entrevistas con sus corredores y administradores, nos ha permitido establecer la geografía de los principales desarrollos impulsados por estos instrumentos durante estos últimos seis años ${ }^{4}$. Nuestras primeras conclusiones confirman el alto grado de selectividad geográfica en sus colocaciones, así como su marcado sesgo metropolitano (Le Fur, 2006). El 95,13\% de las unidades financiadas se concentra en el área metropolitana del Gran Santiago (prácticamente 32.000 viviendas). No obstante, sus efectos se hacen sentir

1 A principios de 2007, la ACAFI -Asociación Chilena de Administradoras de Fondos de Inversión- propuso una nueva clasificación de los fondos en función de su política de inversión. Para este trabajo hemos seleccionado debido a su importancia, a los fondos abiertos que intervienen en la producción de vivienda destinada para la venta (principalmente los Fondos Inmobiliarios de Desarrollo y, parcialmente, los Fondos Inmobiliarios Mixtos).

2 En comparación, el mayor desarrollador de vivienda chileno, Salfacorp s.a.-Aconcagua s.a., no reivindicaba una participación superior al 7,6\% de sus mercados de operación en el ejercicio 2009.

3 Según cifras del Informe del mercado de oficinas de Global Property Solutions, la renta generada por el stock de oficinas de categoría A y A+ en Santiago puede ser estimada a 10,6 millones de U.F. para el año 2010, contra ventas de vivienda nueva por 77,7 millones de U.F. en un mal año.

4 La fiscalización de los fondos de inversión transados en el mercado de valores por la Superintendencia de Valores y Seguros (S.V.S.), prevista por el Art. $1^{\circ}$ de la Ley $n^{\circ} 18.815$ del 29 de julio de 1989, y la oferta inicial de cuotas en el mercado publico permiten acceder con relativa facilidad a la información financiera de estos dispositivos y explicar, por ende, su elección como indicador para el análisis de las estrategias de inversión inmobiliaria de los agentes financieros. Debemos señalar que la información sobre el portafolio de inversiones varía en su precisión entre las diferentes administradoras de fondos de inversión y según el tipo de activos. Si se disponen de datos fiables y prácticamente exhaustivos para los desarrollos inmobiliarios, la información sobre inversiones de renta y de plusvalía es fragmentaria. 
de manera desigual en el territorio metropolitano. Si su rol en la suburbanización residencial y en la gama de vivienda más onerosa parece modesto, su influencia en el fenómeno de la verticalización y en la renovación de los barrios céntricos es preponderante. Como veremos, estos arbitrajes sobre la localización de la inversión dependen de estrategias de atenuación del riesgo basadas en la teoría moderna de la selección de cartera (Markowitz, 1952) y de las características estructurales de cada fondo (plazo de duración, tipo de aportantes). Para Ingrid Nappi-Choulet (1999), esta propagación de las lógicas e imperativos financieros al conjunto de las actividades urbanas conlleva la fabricación de nuevos paisajes urbanos y de un nuevo modelo de ciudad "financiarizada". La metrópolis no es tan solo un centro de articulación de las redes del capitalismo global. Materialmente, la fabricación de sus espacios es uno de los mecanismos esenciales de la valorización del capital.

\section{La transformación del mercado de capitales y la evolución de las modalidades de financiamiento de la actividad inmobiliaria}

La primera generación de reformas adoptadas por el régimen militar (1974-1981) tuvo como objetivo la transformación del marco regulatorio y de la estructuración del sistema bancario, por medio de la liberalización de las tasas bancarias y de la privatización de sus principales instituciones. Después de las consecuencias desastrosas de la crisis de 1981, una segunda salva de reformas prefirió perfeccionar los dispositivos de supervisión, y promover un mercado de capitales diversificado, animado por instituciones no bancarias (Arrau, 1997). En el mes de octubre de 1981, dos textos fundamentales fueron aprobados, la Ley n ${ }^{\circ} 18.045$ de mercado de valores, que normaba la emisión de valores de oferta pública, y la Ley n ${ }^{\circ} 18.046$ sobre sociedades anónimas, que redefinía las reglas de funcionamiento de las sociedades anónimas abiertas. Las modificaciones siguientes buscaron ampliar paulatinamente el abanico de instrumentos financieros elegibles en el mercado chileno. A este respecto, la disposición más importante para el análisis de la interacción entre mercado de capitales y desarrollo urbano es la creación de los fondos de inversión con cuotas transables en 1989, y la autorización de invertir en sociedades anónimas destinadas al negocio inmobiliario o partícipes de concesiones de obras de infraestructura en uso público ${ }^{5}$. No obstante, durante este primer periodo, la influencia del mercado de capitales sobre el sector de la construcción se hizo sentir sobre todo de manera indirecta, a través de la adquisición masiva de las letras hipotecarias emitidas por los diversos bancos nacionales.

En efecto, el desarrollo del sistema financiero local no sería comprensible si no mencionásemos la adopción en 1980 del principio de la capitalización individual como mecanismo de financiamiento del sistema de pensiones. La creación de las administradoras de fondos de pensiones (A.F.P.) permitió solventar dos de las falencias históricas del mercado de capitales nacional: un ahorro de largo plazo modesto y una canalización insuficiente de los recursos hacia la inversión real (Uthoff, 2001).

Incisos $12^{\circ}$ y $14^{\circ}$ del artículo $5^{\circ}$ de la Ley n ${ }^{\circ} 18.815$ del 29 de julio de 1989. 
Una fracción importante de los capitales colectados de esta manera fue destinada a operaciones de compra de letras hipotecarias, haciendo de estos actores un pilar esencial del sistema de financiamiento de la vivienda privada en Chile. En 1983, la cartera de inversiones de los fondos de pensiones estaba compuesta en un 42,9\% de letras hipotecarias, y durante la década siguiente, este ítem representaba aún entre el 14 y el $16 \%$ de su portafolio 6 . Se estima, de esta manera, que el sistema financiero en su conjunto financió, por medio de la adquisición de letras y mutuos hipotecarios endosables, más del $60 \%$ de la inversión privada en vivienda a principios de los años ochenta y que su contribución alcanzaba todavía un 44\% a principios de la década del 2000 (Morandé y García, 2004). Pero el volumen de los fondos manejados por los fondos de pensiones reclamó rápidamente una mayor diversificación de los activos, y el diseño de vehículos de inversión en nuevos sectores. La promulgación de la Ley de Fondos de Inversión en 1989 respondió a esta exigencia, estableciendo el marco normativo para la creación y el funcionamiento de fondos mobiliarios, inmobiliarios y de capital de riesgo.

Hasta ese entonces, la participación de los fondos de pensiones en el área inmobiliaria había sido prohibida por las autoridades reguladoras, ya que se temían eventuales conflictos de intereses con los afiliados, así como la dispersión de las administradoras en estas actividades conexas (Morandé y García, 2004). La modificación del marco normativo, y la consiguiente posibilidad de acceder al mercado del suelo y de la vivienda urbana fue, por ende, acogida con interés por los inversionistas institucionales. Cinco de los diez fondos creados durante los primeros tres años de la reforma fueron destinados a esta actividad. El primero de ellos, el fondo inmobiliario Aetfin-Mixto, inició sus operaciones en abril de 1991 como parte de la estrategia de diversificación del grupo de capitales norteamericanos Aetna. En 1981, el Banco de Chile había propuesto un joint-venture para adquirir parte de su experiencia internacional en el rubro previsional y de seguros, en vistas de la gestión de una nueva administradora de fondos de pensiones. Pero tras la crisis bancaria de 1982-1985, el grupo Aetna se encontró a la cabeza de dichos establecimientos (Kline, 1992). Su conocimiento anterior del mercado norteamericano, aunado a la necesidad de valorizar los recursos captados por su fondo de pensiones, despertó su interés por un mercado inmobiliario en pleno desarrollo, creando así una antena hipotecaria (Aetna Crédito Hipotecario S.A.) en 1987, y dotándose de un vehículo propio para la inversión inmobiliaria pocos años después. La estrechez de los lazos entre fondos de pensiones y la primera generación de los fondos de inversión inmobiliaria ${ }^{7}$ explica así la importancia del sector de las A.F.P. en su capital. Aún hoy, con una inversión de poco más de medio millar de dólares en estos instrumentos, los fondos de pensiones son sus principales contribuyentes, repartiéndose el $72 \%$ de sus cuotas ${ }^{8}$.

6 Fuente: servicio estadístico de la Superintendencia de Pensiones.

7 Fondo de Inversión Inmobiliaria Cimenta-Expansión (20 de mayo de 1991), Toesca Fondo de Inversión, anteriormente Fondo de Inversión Aetfin-Mixto (17 de septiembre de 1991), Fondo de Inversión Inmobiliaria Santander Mixto (27 de noviembre de 1991), Fondo de inversión Las Américas-Raíces (4 de junio de 1992), Fondo de Inversión Santander Plusvalía (31 de marzo de 1992).

8 Fuente: Superintendencia de Pensiones, diciembre 2009. 
Durante este primer periodo, la política de inversión de estos instrumentos fue bastante diversificada, combinando la participación minoritaria en el capital de sociedades inmobiliarias, la adquisición de bienes comerciales y de oficinas - generalmente de gama intermedia- para su arriendo y la compra de terrenos en la periferia de la ciudad para la captura de plusvalías. Los proyectos residenciales fueron un área prospectada, pero se prefirió la focalización en un número limitado de desarrollos de gran importancia. La actividad inmobiliaria del fondo Cimenta-Expansión se concentró, por ejemplo, en el desarrollo de programas habitacionales de varias decenas de hectáreas y con un horizonte de programación de diez o más años. En Lo Barnechea, el inicio del loteo Jardines del Sol data de 1995, y todavía existían productos en venta en 2009. Su segunda gran operación, Jardines de la Viña en La Florida (2001) se hallaba ese mismo año en su octava etapa de desarrollo. En cierto modo, la estrategia de comercialización de estos proyectos los asemeja a inversiones de tipo renta, ya que la urbanización progresiva por etapas anuales permite la obtención de un flujo de caja periódico y a largo plazo. Este mix de actividades reveló ser eficaz, y sus balances fueron holgadamente positivos gracias al dinamismo de la construcción durante la primera mitad de la década de los noventa.

La embestida de la onda depresiva de la "crisis asiática" puso término al ciclo de crecimiento del sector inmobiliario y aumentó drásticamente las tasas de vacancia de los espacios comerciales y de las oficinas corporativas. El impacto sobre la rentabilidad de los fondos inmobiliarios existentes fue consecuente. El lapso 1996-2004 sólo conoce la creación de un nuevo vehículo de inversión. En cambio, durante el periodo siguiente, la inercia del sector fue rota gracias a cambios en la estrategia de las administradoras de fondos de inversión, a la aprobación de nuevas figuras legales y a la reactivación del mercado inmobiliario. En apenas cuatro años (20042007), el mercado de valores local fue reanimado por el establecimiento de once nuevos fondos inmobiliarios. Esta nueva camada de fondos se distingue netamente de la estrategia de colocaciones y de los objetivos de los instrumentos ofertados durante la etapa anterior. Su grado de especialización es mucho más importante, abocándose casi exclusivamente al financiamiento de desarrollos residenciales de primera vivienda. La duración prevista es mucho más breve, variando entre cinco y diez años, cuando los plazos previstos por los fondos de la generación anterior oscilaban entre los veinte y los cuarenta años. Pero la diferencia más importante concierne sin lugar a dudas la estrategia de sus colocaciones. La participación de los inversionistas institucionales es netamente minoritaria y, a veces, inexistente. Desde el momento de su concepción, esta nueva generación de fondos inmobiliarios fue destinada a inversionistas "particulares", con aportes menos importantes, pero mucho más numerosos. Los capitales así reunidos han hecho de este tipo de fondos una de las principales fuentes de financiamiento de la inversión privada en vivienda en el área metropolitana de Santiago. 
FIgURA 1 | Patrimonio (en millones de dólares) de los fondos de inversión inmobiliarios según tipo de fondo.

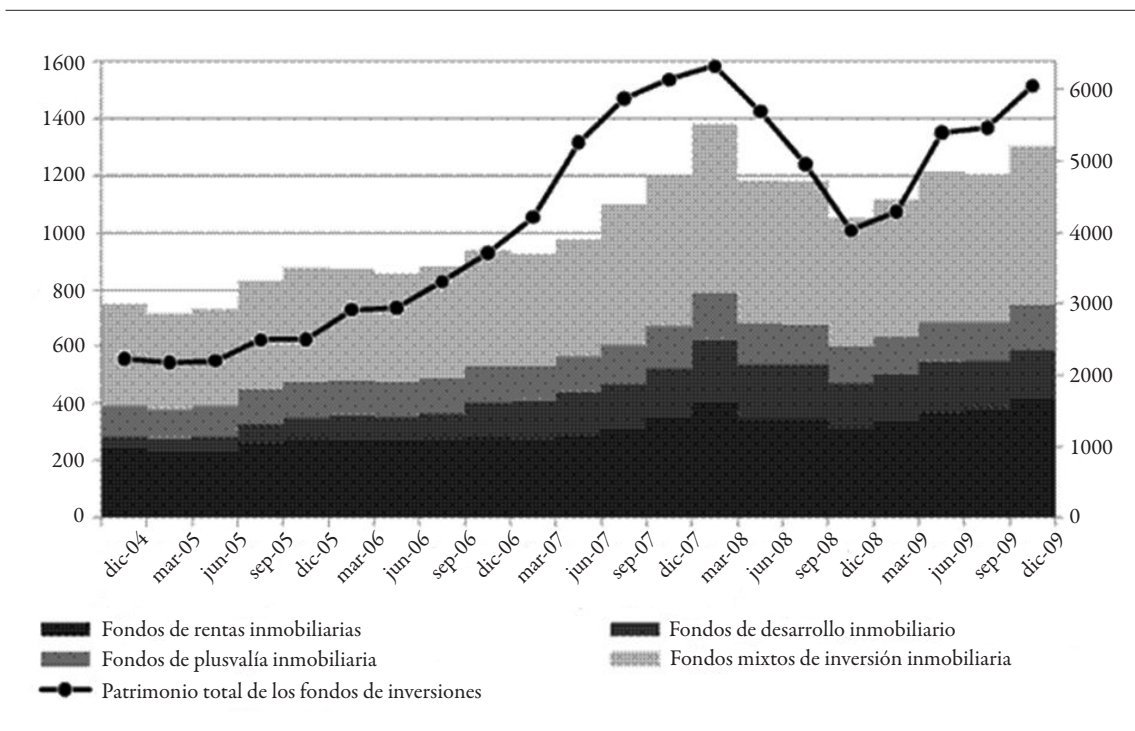

Fuente Cálculos propios en base a ACAFI - Asociación Chilena de Administradoras de Fondos de INVERSIÓN.

En efecto, la década del 2000 ha sido un periodo fructífero para la inversión financiera e inmobiliaria en Chile. Tanto los montos invertidos como el patrimonio gestionado por los fondos inmobiliarios han aumentado rápidamente durante este decenio (Figura 1). En diciembre de 2004, el patrimonio total de los fondos de inversión sobrepasaba difícilmente los dos millares de dólares. Tres años después, este monto se veía casi triplicado. El patrimonio administrado por los fondos inmobiliarios progresó más lentamente. De hecho, su contribución relativa disminuyó de un 34 a un $22 \%$ en el espacio de cinco años. Pero no debemos olvidar que esto aún representa una saludable duplicación del valor total de los bienes urbanos poseídos por estos actores. Su influencia en el sector habitacional se ha reforzado, sobre todo si consideramos que la edificación escapa a las estadísticas patrimoniales. La mayor parte de los fondos que ha declarado recientemente su inicio de actividades corresponde a la categoría de los fondos inmobiliarios de desarrollo, o sea, dispositivos especializados en la inversión y en el desarrollo de vivienda para la venta. Podemos estimar que sus colocaciones han impulsado obras por casi 21.000 unidades en el área metropolitana de Santiago (Figura 2), equivalentes al 8,7\% del total de permisos de edificación emitidos en la capital para el periodo 2004-2008. Los vehículos de inversión mixtos también han contribuido a este verdadero boom inmobiliario, asociándose a la construcción de otras 10.000 viviendas (Figura 2). Los fondos de inversión inmobiliarios son hoy en día uno de los agentes clave en el sistema de producción de la ciudad. En total, el mercado de capitales ha financiado a través 
de estos dos tipos de instrumentos un $13,8 \%$ de la vivienda privada construida en Santiago y ha hecho posible ventas por 65 millones de UF.

FIGURA 2 La contribución de los fondos de inversión inmobiliaria a la construcción habitacional privada en Santiago, 2004 - 2009.

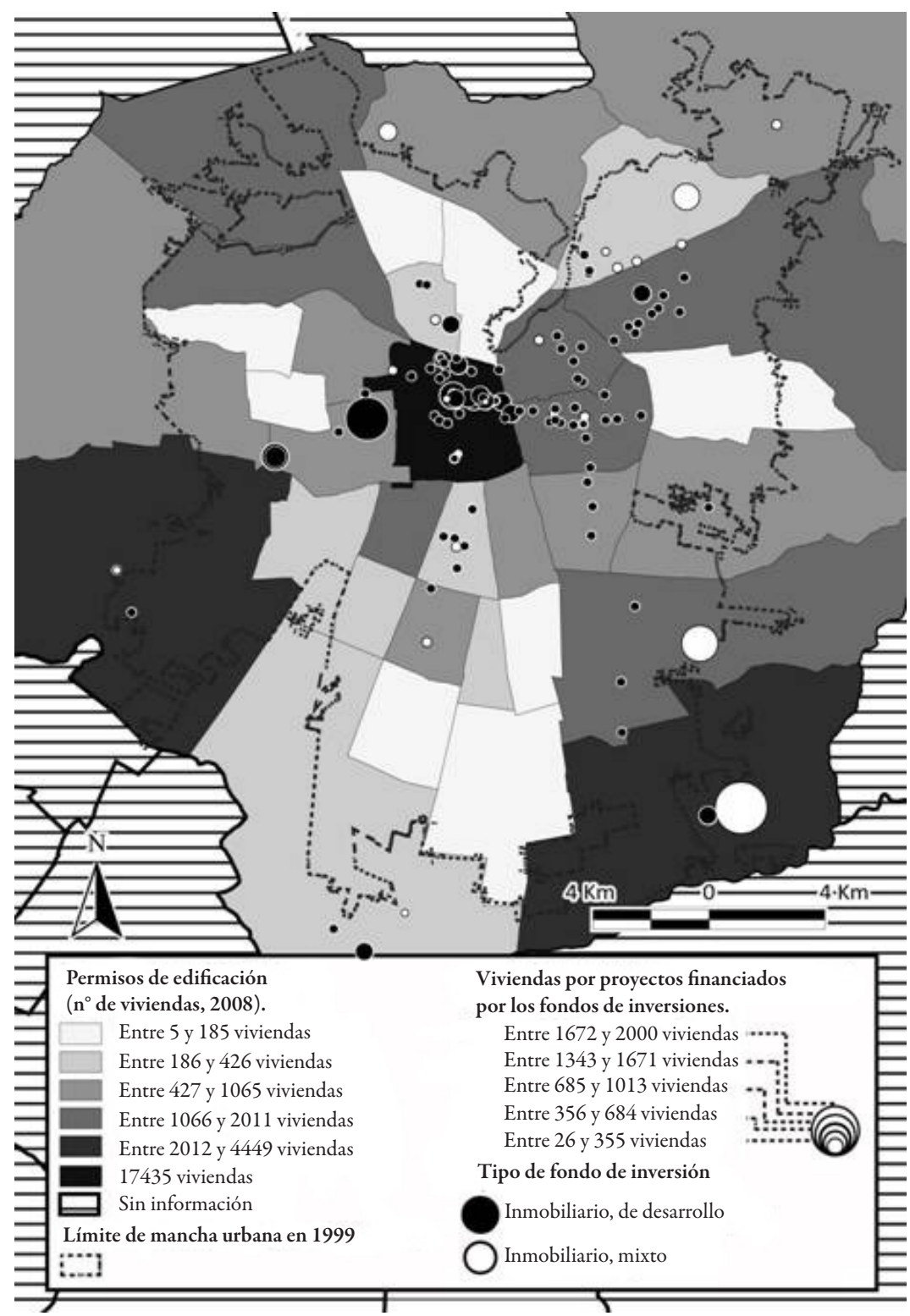

FUENTE ELABORACIÓN PROPIA EN BASE A LAS MEMORIAS DE ACTIVIDADES ANUALES Y A LA ESTADÍSTICA DE EDIFICaCión de la Cámara Chilena de la Construcción. 
Un paralelo puede ser establecido entre las circunstancias que vieron el nacimiento de estas dos generaciones de fondos de inversión. En ambos casos, se destaca la importancia de las transformaciones estructurales del mercado financiero y el rol clave jugado por agentes internacionales en su diseño inicial. En particular, la administradora ligada al banco de capitales españoles Santander fue un actor central de este proceso. El lanzamiento de su primer fondo de inversiones de desarrollo inmobiliario importó a Chile el modelo, ya probado en España, de captación de recursos de particulares para el desarrollo de programas residenciales destinados a la venta. En una coyuntura de alto dinamismo de la construcción, el éxito de esta primera operación fue contundente y justificó el establecimiento paulatino de seis nuevos fondos por esta misma administradora. La consolidación del sector bancario chileno y la importancia de su actividad de gestión patrimonial estimularon también la exploración de nuevas opciones de diversificación. No es sorprendente que once de los dieciséis fondos de este tipo sean controlados por administradoras de origen bancario, y que cada uno de ellos cuente con un promedio de 103 aportantes diferentes.

La importancia adquirida por estos grupos en el financiamiento de la ciudad ha tenido consecuencias significativas sobre la estructuración de la rama de la construcción. Tanto en Chile como en el resto del mundo, el sector inmobiliario se caracteriza por su fragmentación (OECD, 2008). Pero, para la realización de sus proyectos, los fondos de inversión no se asocian a empresas inmobiliarias de manera puntual o aleatoria. Prefieren al contrario desarrollar relaciones duraderas de colaboración. Este modelo de relación ha primado desde un principio. Hasta su compra por Socovesa s.a. en el 2007, la Inmobiliaria Almagro s.a. fue uno de los operadores más importantes del mercado de la vivienda privada de Santiago. Fundada en 1977, desarrolló con éxito sus primeras obras, destinadas a segmentos medios y medios altos, en las comunas de Las Condes y de Nuñoa. Sin embargo, la crisis económica de 1982 la afectó duramente, enlenteciendo su desarrollo. El establecimiento de relaciones financieras y estratégicas con el Fondo Inmobiliario Aetfin-Mixto suscitó una nueva fase de crecimiento para la compañía, que se vio reforzada por la entrada en su capital del grupo Aetna en 1996 y por su introducción en la bolsa dos años después. Relaciones similares entre fondos y promotores pueden ser identificadas hoy en día. Cuarenta y cuatro gestores inmobiliarios intervienen en los 124 programas habitacionales en los que hemos podido identificar el conjunto de los participantes, pero los diez más importantes aglutinan la mitad de estos contratos. Este modelo de intervención en el mercado de la vivienda santiaguino ha favorecido la emergencia y la consolidación de una capa de empresas de medianas dimensiones, con una producción anual que oscila en torno de las quinientas viviendas anuales, y especializadas en la construcción y venta de programas habitacionales en condominios. El dinamismo de los fondos de inversión es más que una simple fuente alternativa de financiamiento de la vivienda, es una de las fuerzas que reconfigura al conjunto de este sector. 


\section{La financiarización de la ciudad: estrategias espacializadas de construcción de portafolio, periodización de la inversión y nuevos paisajes urbanos}

Con la publicación de Limits to capital, David Harvey (1992) reafirmó la importancia del factor territorial de los procesos productivos -tanto materiales como inmateriales- y reintrodujo así la problemática geográfica en el análisis de la economía. Según Harvey, la expansión hacia nuevas regiones y la reorganización espacial de sus bases productivas son dos momentos clave para la superación de las contradicciones internas del mercado. Estas hipótesis fueron fundamentales para la teorización de las interacciones entre sistema económico y formas espaciales. La transformación de la metrópolis era tanto una necesidad para que pudiesen jugar eficazmente su rol de plataforma de las lógicas expansivas del capitalismo, como uno de los múltiples vehículos de valorización del capital (Brenner y Theodore, 2002). En vez de contradecirse, la suburbanización, con su seguidilla de loteos periféricos, y la recuperación de los espacios centrales pueden entonces ser interpretadas como dos momentos sucesivos y complementarios de la circulación del capital en los espacios metropolitanos (Smith, 2002). A este respecto, los instrumentos de inversión financiera han probado ser en Chile un agente reestructurador del sistema de producción de la ciudad. Nuestro análisis del portafolio de los activos habitacionales de los fondos de desarrollo revela, en efecto, un marcado sesgo metropolitano y un alto grado de selectividad en la implantación de estos proyectos. Pero sus impactos sobre la organización espacial de Santiago son mucho menos unívocos. Los fondos de inversión, y en especial los fondos de desarrollo inmobiliario, son indudablemente uno de los motores de la verticalización de la ciudad y de la renovación de los barrios centrales. En cambio, la evidencia es menos concluyente sobre su rol en la extensión periférica de la capital y descarta un proceso de "elitización" impulsado por el capital financiero.

Tomados uno a uno, el impacto de los fondos de desarrollo inmobiliario sobre la metrópolis parecería ser modesto. Cada uno de ellos ha financiado, a una tasa siempre igual o inferior al 50\%, un promedio de 7,5 proyectos, de una talla media de 196 viviendas, y dispersos entre 4 y 5 comunas. En cambio, el análisis de la implantación de la totalidad de estos proyectos devela algunos de los circuitos de valorización del espacio urbano. El sesgo metropolitano en la delimitación de la cartera de inversiones es una primera evidencia. El 97,88\% de la vivienda financiada se localiza en la Región Metropolitana, y se podría hablar de su totalidad si tomásemos en cuenta el área de influencia de la metrópolis ${ }^{9}$. Los fondos mixtos presentan resultados apenas más equilibrados para esta categoría de bienes (94,1\% en la Región Metropolitana), pero la repartición de sus bienes raíces destinados a la renta y a la realización de futuras plusvalías tiende a una mayor dispersión, con una presencia apreciable en las capitales regionales del país (Concepción, Antofagasta). El carácter selectivo de la inversión inmobiliaria es también perceptible en valorización desigual de los espacios intrametropolitanos. El Cuadro 1 indica la distribución de los bienes 
producidos por estos inversionistas. Si veinte comunas de la Región Metropolitana acogen por lo menos un proyecto, el $90 \%$ del total construido se concentra en diez comunas, y casi el $75 \%$ en las cinco primeras. Por supuesto, estas desigualdades reproducen en parte las disimetrías del mercado local. Estas diez comunas concentraban ya el 65\% de los permisos de edificación acordados en Santiago. No obstante, los criterios de localización que arbitran estas implantaciones develan otras lógicas, y particularmente una evaluación diferenciada del valor de los territorios comunales según las exigencias de diferentes tipos de inversionistas y de instrumentos.

CUADRO 1 | Operaciones residenciales de los fondos de desarrollo inmobiliario y mercado de la vivienda en Santiago

\begin{tabular}{|c|c|c|c|c|c|c|c|}
\hline \multirow[b]{2}{*}{ Comuna } & \multicolumn{3}{|c|}{$\begin{array}{c}\text { FONDOS INMOBILIARIOS DE } \\
\text { DESARROLLO }\end{array}$} & \multicolumn{2}{|c|}{$\begin{array}{c}\text { PERMISOS DE } \\
\text { EDIFICACIÓN - SANTIAGO } \\
(2004-2008)\end{array}$} & \multicolumn{2}{|c|}{$\begin{array}{l}\text { MERCADO HABITACIONAL } \\
(2008)\end{array}$} \\
\hline & $\begin{array}{l}\mathrm{N}^{\circ} \mathrm{DE} \\
\text { VIV. }\end{array}$ & $\begin{array}{c}\text { VIV. } \\
(\mathrm{EN} \%)^{\mathrm{A}}\end{array}$ & 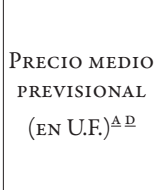 & $\begin{array}{l}\text { Permisos } \\
(\text { EN } \%)^{\underline{B}}\end{array}$ & $\mathrm{RANGO}^{\underline{B}}$ & $\begin{array}{c}\text { PRECIO MEDIO } \\
\text { OBSERVADO } \\
\text { (VIVIENDA, EN } \\
\text { U.F. })^{\mathrm{C}}\end{array}$ & $\begin{array}{c}\text { RELACIÓN } \\
\text { ENTRE LOS } \\
\text { PRECIOS } \\
\text { PREVISIONALES } \\
\text { (F.I.) Y } \\
\text { OBSERVADOS CE }\end{array}$ \\
\hline Santiago & 9701 & $46,72 \%$ & 1393 & $27,62 \%$ & 1 & 1610 & $-13,48 \%$ \\
\hline Ñuñoa & 1755 & $8,45 \%$ & 2809 & $7,21 \%$ & 5 & 2734 & $2,74 \%$ \\
\hline Las Condes & 1508 & $7,26 \%$ & 4085 & $7,28 \%$ & 4 & 4706 & $-13,20 \%$ \\
\hline San Miguel & 1265 & $6,09 \%$ & 1914 & $1,03 \%$ & 18 & 2237 & $-14,44 \%$ \\
\hline Independencia & 1084 & $5,22 \%$ & 1327 & $1,50 \%$ & 16 & 1517 & $-12,52 \%$ \\
\hline Macul & 896 & $4,31 \%$ & 2196 & $2,07 \%$ & 13 & 2047 & $7,28 \%$ \\
\hline Providencia & 868 & $4,18 \%$ & 2943 & $5,44 \%$ & 6 & 3351 & $-12,18 \%$ \\
\hline Recoleta & 568 & $2,74 \%$ & 1712 & $2,64 \%$ & 8 & 1747 & $-2,00 \%$ \\
\hline Puente Alto & 488 & $2,35 \%$ & 1705 & $7,86 \%$ & 3 & 2280 & $-25,22 \%$ \\
\hline San Bernardo & 482 & $2,32 \%$ & 1011 & $2,23 \%$ & 11 & 1812 & $-44,21 \%$ \\
\hline
\end{tabular}

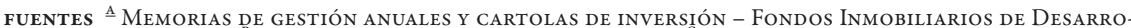
LLO, AÑos 2004 - 2008 B CÁMARA CHILENA DE LA CONSTRUCCIÓN ${ }^{-}$ESTIMACIONES PROPIAS EN BASE A DECON, PORTAL INMOBILIARIO (WWW.PORTALINMOBILIARIO.COM).

NOTAS D LA COLUMNA "PRECIO MEDIO PREVISIONAL" CORRESPONDE A UNA ESTIMACIÓN DEL PRECIO DE VENTA DE LOS BIENES CONSTRUIDOS, TAL COMO FUERON COMUNICADA A LOS APORTANTES DE LOS FONDOS INMOBILIARIOS. En los casos de Macul, Providencia, Recoleta, Puente Alto y San Bernardo, el bajo número de proYECTOS PUEDE EXPLICAR LA MAYOR VARIACIÓN DE LOS PRECIOS.

En primer lugar, podemos notar que la actividad de los fondos inmobiliarios ha favorecido espectacularmente la construcción en altura. En 2008, el 22,6\% de los permisos de edificación correspondía a viviendas de uno o dos pisos. En cambio, las casas representaban menos del $9 \%$ de la vivienda prevista por los operadores financieros. La distinción entre estas dos categorías de bienes explica en parte la configuración de su cartera de inversiones. A pesar de formar parte del quinteto de las co- 
munas con el mayor número de permisos de edificación otorgados en 2008, Puente Alto, Maipú, La Florida o Renca representan opciones insignificantes o marginales para estos fondos, ya que los mercados locales se han especializado en la comercialización de lotes y casas individuales. Su contribución al lanzamiento de los grandes megaproyectos periféricos (Piedra Roja, Valle Grande) parece también haber sido muy modesta. Estaríamos de este modo frente a una modalidad alternativa a la "ciudad vallada” (Borsdorf, Hidalgo y Sánchez, 2007) de valorización del espacio urbano. El centro histórico de Santiago ha sido en cambio objeto de una prolongada política de renovación basada en el subsidio a la demanda y en el favorecimiento de la acción de las empresas de construcción por medio de una norma, inicialmente, permisiva (Paquette, 2004). Este proceso promovió la reanimación del mercado comunal y la construcción de numerosos condominios verticales. Con el $31 \%$ de sus operaciones y el $47 \%$ de la vivienda financiada localizada en este perímetro, los fondos inmobiliarios de desarrollo son uno de los motores de este proceso y de su extensión a comunas aledañas. Independencia y San Miguel representan, en efecto, el $11 \%$ de sus colocaciones. Pero este monto es sobre todo significativo si se le compara con la actividad inmobiliaria general de estos espacios. Con 1.265 y 1.084 departamentos, su participación en el mercado de San Miguel y de Independencia equivale respectivamente al $52 \%$ y al $30 \%$ del número de permisos concedidos en estas comunas para el periodo 2004-2008.

La Figura 3 aporta ciertas luces para entender el porqué de estos arbitrajes. Este mapa indica la localización de los desarrollos inmobiliarios para todo tipo de fondos, así como la estimación de sus ventas. Podemos así constatar que los fondos de tipo mixto no han renunciado a una actividad en la periferia de la ciudad. Sus proyectos más importantes, tanto en términos de ventas como de viviendas construidas, se hallan en estos espacios. En cambio, los programas residenciales de los fondos de desarrollo privilegian una localización central y la proximidad de los principales ejes estructurales de la ciudad. La diferenciación de sus estrategias puede ser explicada por el desfase entre el ritmo de funcionamiento de los fondos de inversión y el desarrollo de las urbanizaciones horizontales. La duración de los fondos de desarrollo inmobiliario, generalmente de cinco años, implica plantearse desde el principio el problema de la liquidación de sus activos. El ritmo más rápido de concepción, construcción y comercialización de un proyecto inmobiliario en altura, inferior a dos años, corresponde al ritmo de marcha de esta categoría de fondo. Al contrario, la necesidad de abordar operaciones preliminares de urbanización, así como el modelo de desarrollo y venta en etapas sucesivas de los proyectos habitacionales horizontales se adapta mejor a la exigencia de ingresos regulares y duraderos de los inversionistas institucionales, y a los horizontes de mediano plazo de fondos inmobiliarios mixtos. El mercado de suelo, pero siempre en relación al problema de los ritmos de inversión y de retorno, es otro factor de explicación de esta situación. El carácter oligopólico de la estructura de propiedad del suelo en la periferia, la expectativa de importantes plusvalías futuras y la importancia de las reservas de terrenos ya adquiridas por empresas inmobiliarias favorece la retención 
del suelo por sus propietarios. En un artículo reciente sobre las redes empresariales en el sector inmobiliario, Gloria Yáñez, Johannes Rehner y Oscar Figueroa (2010) recalcan que la propiedad de la tierra es un recurso de poder considerable en la constitución de nuevas alianzas de actores inmobiliarios y que los vínculos personales son muchas veces esenciales para sortear desacuerdos. Este marco de negociación desfavorece, por ende, el ingreso al mercado periférico de los instrumentos financieros, creaciones recientes y de origen generalmente bancario.

FIGURA 3 | Ventas estimadas según tipo de fondo de inversión inmobiliaria, 2004-2009

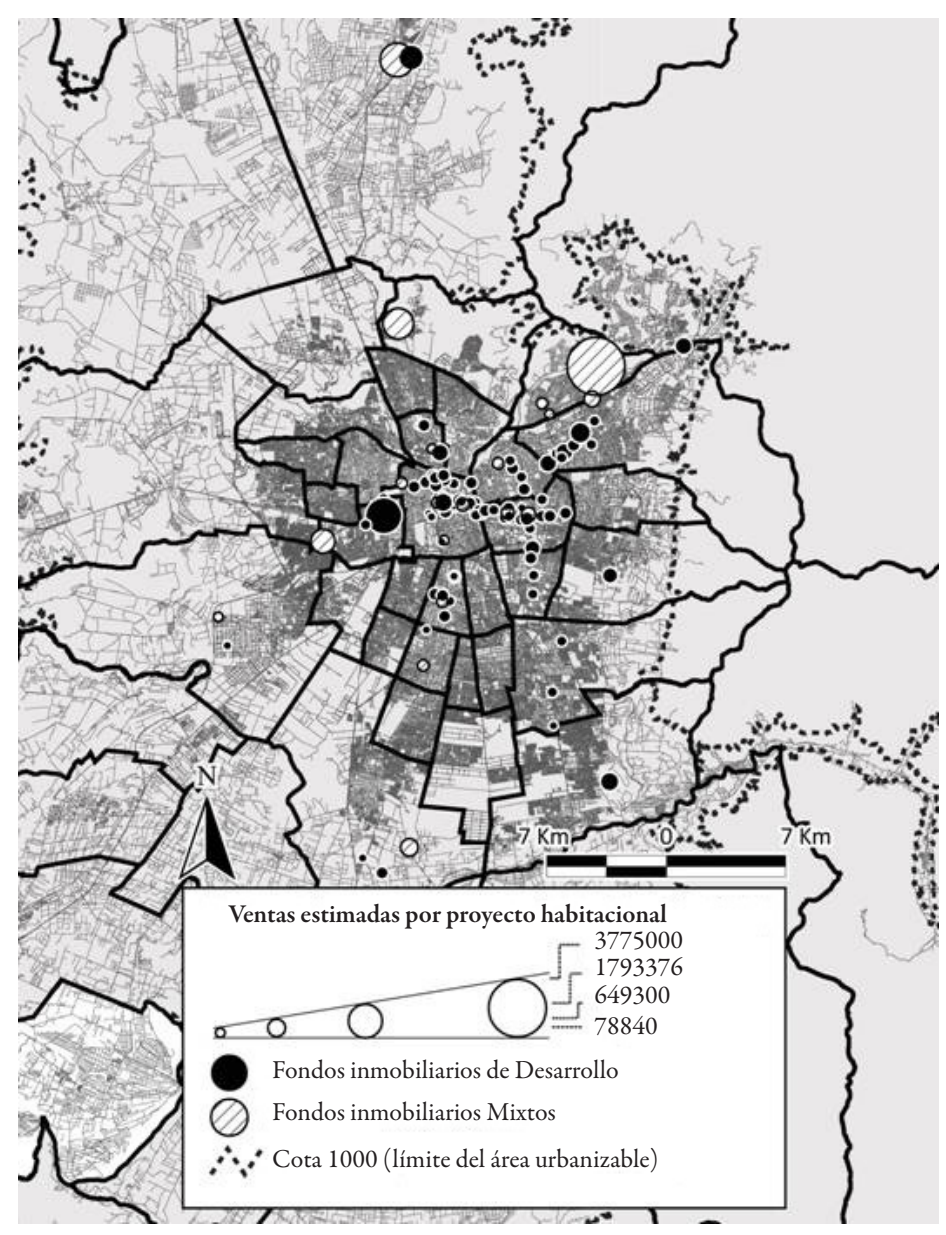

FUENTE ELABORACIÓN PROPIA EN BASE A LAS MEMORIAS DE ACTIVIDADES ANUALES Y A LAS CARTOLAS TRIMESTRALES DE PROYECTOS. 
La adecuación entre el horizonte de funcionamiento de los fondos inmobiliarios de desarrollo y el tiempo de programación de una operación residencial en altura explica la concentración de su inversión en áreas centrales, pero es insuficiente para aclarar la distribución entre las diferentes comunas de la ciudad consolidada. Esta geografía altamente selectiva depende tanto de factores de orden sectorial como financiero. Los desarrolladores inmobiliarios son un primer filtro para la constitución de un portafolio de activos. El capital financiero no es ubicuo. Los costos de adquisición de la información necesaria para el lanzamiento de una operación inmobiliaria exitosa impiden una circulación perfectamente fluida del capital. La entrada en el negocio urbano exige la mediación de actores especializados, dotados justamente de un conocimiento específico del mercado, de su oferta y de su demanda. La asociación privilegiada entre cada un fondo y dos o tres promotores resulta, en consecuencia, lógica. Su primera misión consiste justamente en aportar una cartera de proyectos. Pero en un segundo momento, un estudio financiero resulta necesario. El objetivo principal de este análisis no es tanto de definir la viabilidad comercial de cada una de estas operaciones, pero de determinar la combinación más adecuada de inversiones en función de la rentabilidad esperada y de los riesgos que se está dispuesto a asumir. En este proceso, la referencia a la "teoría moderna del portafolio" de Markowitz (1952) es insoslayable. Markowitz constata que el riesgo de un portafolio está determinado por el grado de correlación entre sus activos, y que el riesgo individual de los instrumentos disminuye a medida que aumenta su número. Un portafolio eficiente se define, entonces, como la combinación de instrumentos que ofrece el mejor rendimiento posible para un mismo nivel de riesgo. Estos postulados culminaron en dos grandes tendencias en vistas de la optimización de fondos altamente especializados. En primer lugar, la selección de una categoría de activos o de un mercado como el componente principal -core holding- de un instrumento financiero permite definir su estrategia de inversión y el perfil de riesgo/rendimiento esperado. En segundo lugar, la diversificación de los activos por medio de la dispersión geográfica de la inversión es ineludible para amortizar la volatilidad de los diferentes submercados (Brueggeman y Fischer, 2004).

Estos criterios hacen más legible el patrón de distribución espacial de los proyectos inmobiliarios: una concentración considerable en un pequeño núcleo de comunas con un mercado ya consolidado, y especialmente el casco histórico de Santiago, acompañada de un alto número de proyectos más o menos aislados en otras comunas. No obstante, el criterio de selección del principal perímetro de inversión puede sorprender. Pocos bienes en el caso de los fondos de desarrollo (2.438 viviendas) fueron localizados en el "cono de altos ingresos" de Santiago, uno de los submercados más importantes por los montos transados (con menos de un 10\% de la vivienda privada construida en 2008, las comunas de Providencia, Las Condes, Lo Barnechea y Vitacura concentraban el $29 \%$ de la oferta inmobiliaria en valor ${ }^{10}$. En cambio, si se compara la distribución de las inversiones de los fondos de desarrollo (Cuadro 1) y el número de permisos de edificación acordados en el área metropoli-

Estimaciones propias en base a Decon y Portal Inmobiliario (www.portalinmobiliario.com). 
tana de la capital, se puede identificar un patrón común en la sobrerrepresentación de las comunas de áreas centrales - Santiago y sus comunas aledañas de San Miguel, Independencia, Nuñoa y Macul- y en el tipo de bien propuesto -situados en un rango de 1.500 a 2.700 unidades de fomento. Contrariamente al caso de Buenos Aires (Baer, 2008), el sector inmobiliario privado no ha privilegiado la vivienda "premium" sino que se ha volcado hacia la producción habitacional de gamas intermedias. En la mayoría de los casos, se trata de departamentos de dimensiones pequeñas en inmuebles de mediano standing, destinados a la accesión a la propiedad de hogares unipersonales de jóvenes profesionales o de parejas sin hijos.

La utilización de nuevas herramientas de segmentación y análisis de la demanda (geo-marketing, estudios de mercado) había permitido identificar cambios importantes en la estructura de la demanda habitacional (Cattaneo, 2010). La reestructuración de la economía metropolitana favoreció el aumento de las capas técnicas y profesionales, una mejor integración al mercado del trabajo formal y una mejora substancial de sus ingresos (De Mattos et al., 2005). La confluencia entre este fenómeno, el acceso simplificado al crédito, y el aval estatal aportado al desarrollo de los espacios centrales por medio del subsidio de interés territorial desembocó en un aumento considerable de la inversión. Entusiasmados por las perspectivas de crecimiento de este nicho y por la velocidad más rápida de salida de los productos (al menos durante los primeros años), la apuesta espacial de los fondos de desarrollo inmobiliario fue la profundización de este mercado de accesión a la propiedad, con una intervención importante en la comuna de Santiago, y la exploración de zonas adyacentes con características similares en la búsqueda de una mayor diversificación. El análisis de los precios de venta de sus productos confirma esta intuición (Cuadro 1). Aunque esta variable debe ser manipulada con precaución, ningún elemento permite concluir la responsabilidad de los inversionistas financieros en la "elitización" de los espacios centrales. Es más, los precios de venta comunicados se hallan por lo general bajo la media de los valores observados en estas mismas comunas. La estrategia de estos vehículos financieros parece ser antes que nada la aceleración del ciclo de capital de trabajo. En vistas de este objetivo, han favorecido las formas -condominios habitaciones en altura, departamentos de uno o dos ambientes-, los espacios -las comunas centrales- y las políticas de precios más adecuadas a la captura del segmento más reciente y dinámico de la demanda inmobiliaria, las categorías de ingresos intermedios.

\section{Conclusión}

El comportamiento del mercado habitacional metropolitano revela la influencia creciente de las decisiones y de los arbitrajes de los operadores del mercado de capitales sobre este sector. Chile no ha sido extranjero a la tendencia mundial a la "financiarización" de los circuitos inmobiliarios. Producida industrialmente y administrada según lógicas patrimoniales, la vivienda entraba de manera muy excepcional en el portafolio de activos de los inversionistas internacionales hasta mediados de 
los años ochenta. Desde ese entonces, la flexibilización de los marcos normativos de inversión, la adopción de regímenes fiscales más favorables y la búsqueda de una mayor diversificación por los inversionistas institucionales favorecieron una extensión del perímetro de actividad financiera. Multiplicando las adquisiciones y el lanzamiento de nuevos proyectos, los diferentes vehículos de intervención en el mercado inmobiliario -Real State Investment Trusts anglosajones, Offene Inmobilienfonds alemanes, Sociétés d'Investissement Immobilier Côtées francesas- emergieron como uno de los principales agentes del desarrollo de las metrópolis mundiales (Le Fur, 2006). El término de "financiarización" no sólo consagra estos cambios en la estructura de la propiedad del suelo, sino intenta también traducir las mutaciones inducidas por la participación de esta nueva categoría de actores. El marcado sesgo metropolitano de sus colocaciones, su preferencia por ciertas formas arquitecturales (los rascacielos, el open space terciario, los centros comerciales) y la difusión de técnicas de gestión directamente extraídas de la esfera de la finanza al campo urbano fueron así factores esenciales de la emergencia de la metrópolis "global" (Nappi-Choulet, 1999).

Aunque menos sofisticados y animados preferentemente por capitales locales, los fondos de inversión inmobiliaria jugaron un rol similar, canalizando la disponibilidad creciente de recursos en el mercado financiero chileno hacia la actividad de la construcción. Actuaron así como un formidable acelerador de la producción de vivienda privada y como uno de los motores de la verticalización de los paisajes metropolitanos. Se confirma así la proposición inicial de una articulación entre la nueva fase de modernización capitalista y el advenimiento de un nuevo modelo de ciudad. El rol cada vez más considerable del negocio inmobiliario no estará exento de plantear nuevos desafíos para su gobernanza. La alta selectividad geográfica de la inversión, así como su periodización en función de un "market timing" favorecen un desarrollo desigual de los territorios urbanos y fortalecen las dinámicas de fragmentación (Renard, 2008).

Pero si estas hipótesis son generalmente admitidas, no se les puede reducir en ningún caso a una respuesta definitiva a la cuestión del cómo y del por qué de la transformación de la metrópolis. Su interés reside en su capacidad a proponer una nueva agenda para la investigación académica, incitando un análisis diferenciado de las estrategias espaciales de los diversos agentes de la economía urbana. A su modesta escala, este artículo expone las lógicas disímiles de inversión de los diferentes tipos de fondos inmobiliarios, y subraya su importancia en la creación de un nuevo skyline para la capital y en emergencia de los barrios céntricos como uno de los más activos submercados habitacionales de la capital. En cambio, en la consolidación de los central building districts capitalinos, los capitales internacionales y los inversionistas institucionales parecen haber jugado un rol preponderante. En la periferia, a pesar de que la liberalización económica y la globalización hayan sido señaladas como causas de la suburbanización de las elites y del urban sprawl (De Mattos, 2007; Parnreiter, Fisher y Jäger, 2005), la evidencia empírica revela una 
influencia más bien modesta de los dispositivos estudiados, limitada generalmente a una función de intermediación en las transacciones de suelo (bodegaje de terrenos). Estos ejemplos incitan a continuar el examen de las múltiples modalidades de articulación entre la esfera económica y urbana, ya que los fondos de inversión inmobiliaria son sólo uno de los numerosos vehículos de valorización del capital en la ciudad. Para evitar el escollo de una generalización excesiva, la lectura de los efectos de las transformaciones estructurales debe ser sensible a la diversidad de los sistemas de actores urbanos, al enraizamiento espacial de sus operaciones y a la especificidad de su accionar (Brenner y Theodore, 2002; Peck y Tickell, 2002). IEURE

\section{Referencias bibliográficas}

Arrau, P. (1997). Desarrollo del mercado de capitales: Lecciones de la experiencia chilena. Perspectivas, Vol. 1, No. 1, pp. 25-43.

Baer, L. (2008). Crecimiento económico, mercado inmobiliario y ausencia de política de suelo. Un análisis de la expansión del espacio residencial de la ciudad de Buenos Aires en los 2000. Proyección, Vol. 5, No. 2.

Borsdorf, A. (2003). Cómo modelar el desarrollo y la dinámica de la ciudad latinoamericana. Eure, Vol. 29, No. 86, pp. 37-49.

Borsdorf, A.; R. Hidalgo \& R. Sánchez (2007). A new model of urban development in Latin America: The gated communities and fenced cities in the metropolitan areas of Santiago de Chile and Valparaíso. Cities, Vol. 24, No. 5, pp. 365-378.

Brenner, N. \& Theodore, N. (2002). Cities and the Geographies of 'Actually Existing Neoliberalism'. Antipode, Vol. 34, pp. 349-379.

Brueggeman, W. \& Fischer, J. (2004). Real Estate Finance and Investments. Boston: McGrawHill/Irwin.

Cattaneo, R. (2010). L'A.B.C. du marketing: les dispositifs de stratification sociale et la gouvernance par le marché. En Séminaire ANR Métraljeux, Paris, 9 juin 2010, Enjeux urbains et gouvernance en quatre métropoles d'Amérique Latine : Le Logement.

Cifuentes, R.; Desormeaux, J. \& González, C. (2002). Capital markets in Chile: from financial repression to financial deepening. Economic policy papers, No 4. Santiago: Banco Central de Chile.

De Mattos, C. (2003). Globalización y transformación metropolitana en el caso de Santiago. En F. Arenas, F. Hidalgo \& J. Coll (Eds.). Los nuevos modos de gestión de la metropolización. Santiago: GEOlibros.

De Mattos, C.; Riffo, L.; Yáñez, G. \& Salas X. (2005). Reestructuración del mercado metropolitano de trabajo y cambios socio-territoriales en el Gran Santiago. Santiago: Informe de Investigación Proyecto FONDECYT.

De Mattos, C. (2007). Globalización, Negocios inmobiliarios y transformación urbana. Nueva Sociedad, No. 212, pp. 82-96.

Epstein, G.A. (2005). Financiarization and the world economy, Northampton: Edward Elgar Publishing. Friedmann, J. \& Wolff, G. (1982). World City Formation: An Agenda for Research and Action. International Journal of Urban and Regional Research, No. 6, pp. 309-344. 
Grant, R. \& Nijmann, J. (2002). Globalization and the Corporate Geography of Cities in the LessDeveloped World. Annals of the Association of American Geographers, Vol. 92, No. 2, pp. 320-340.

Hall, P. (1966). The World Cities. London: Weidenfeld and Nicolson.

Harvey, D. (1982). Limits to capital. Oxford: Oxford University Press.

Kline, J. M. (1992). Foreign investment strategies in restructuring economies: learning from corporate experiences in Chile. Westport: Greenwood Publishing Group.

Le Fur, E. (2006). Panorama des fonds immobiliers dans le monde. Management \& Avenir, Vol. 2, No. 8, pp. 87-116.

Markowitz, H. (1952). Portfolio Selection. The Journal of Finance, Vol. 7, No. 1, pp. 77-91.

Meyer, K. \& Bahr J. (2001). Condominios in greater Santiago de Chile and their impact on the urban structure. Die Erde, Vol. 132, No. 3, pp. 293-321.

Morandé, F. \& García, C. (2004). Financiamiento de la vivienda en Chile. Research Department Working Paper, No. 502. New York: Banco Interamericano de Desarrollo (BID).

Nappi-Choulet, I. (1999). Marketing et stratégie de l'immobilier. Paris: Dunod.

OECD (2008). Policy Roundtables : Construction industry. Paris: OECD.

Paquette, C. (2004). La reconquête du centre de Santiago du Chili: un nouveau modèle pour la récupération des centres historiques d'Amérique latine ? Cahiers des Amériques latines, Vol. 2, No. 46, pp. 151-165.

Parnreiter, C.; Fisher, K. \& Jäger, J. (2005). La metrópolis latinoamericana como nodo central en las cadenas productivas globales. Transformaciones socioeconómicas y desarrollo urbano. En R. Hidalgo, R. Trumper \& A. Borsdorf (Eds.). Transformaciones urbanas y procesos territoriales. Lecturas del nuevo dibujo de la ciudad latinoamericana. Santiago: Serie GEOlibros.

Peck, J. \& Tickell, A. (2002). Neoliberalizing Space. Antipode, No. 34, pp. 380-404.

Renard, V. (2008). La ville saisie par la finance. Le Débat, No. 148, pp. 106-117.

Sabatini, F. (2000). Reforma de los mercados de suelo en Santiago, Chile: efectos sobre los precios de la tierra y la segregación residencial. Eure, Vol. 26, No. 77, pp. 49-80.

Smith, N. (1979). Towards a Theory of Gentrification: A Back to the City Movement by Capital, not People. Journal of the American Planning Association, Vol. 45, No. 4, pp. 538-548.

Uthoff, A. (1973). La reforma del sistema de pensiones y su impacto en el Mercado de capitales. En R. Ffrench-Davis \& B. Stallings (Eds.). Reformas, crecimiento y politicas sociales en Chile desde 1973. Santiago, LOM.

Veltz, P. (1996). Mondialisation, villes et territoires. Paris: Presses Universitaires de France.

Velut, S. (2007). Mondialisation et développement territorial en Amérique latine. Université de Paris 3: Habilitation à diriger des recherches.

Yáñez, G.; Rehner, J. \& Figueroa, O. (2010). Redes empresariales e informales en el mercado inmobiliario de Santiago de Chile. Scripta Nova, Vol. 14, No. 331. 